\title{
Patologías físicas sufridas por los profesores de Educación Física de E.S.O de la provincia de Jaén en su lugar de trabajo Physical injuries related to workplace conditions in secondary school Physical Education teachers in Jaen
}

\author{
*María Carrasco Poyatos, **Manuel Vaquero Abellán, ***Alberto Espino Poza \\ *Universidad de Murcia (España), Universidad de Córdoba (España), ***Universiad de Castilla-La Mancha (España)
}

\begin{abstract}
Resumen: La finalidad del presente estudio fue conocer las patologías más frecuentes que afectan a los profesores de Educación Física de E.S.O. de la provincia de Jaén, las bajas que habitualmente les mantienen alejados de su lugar de trabajo y las soluciones que proponen para mejorar su situación laboral y a la vez su salud y su calidad de vida. Participaron 216 docentes de los que se seleccionó al azar una muestra de 50 profesionales. La recogida de datos se llevó a cabo mediante una encuesta original auto cumplimentada. El 30\% de la muestra sufrió alguna patología física en su lugar de trabajo. Las patologías más frecuentes fueron: esguince de tobillo (13\%) y lumbalgia (10\%). La duración de la baja laboral producida por estas patologías fue de 1 a 20 días. Las soluciones que propusieron para reducir la incidencia de lesiones fueron: mejorar los materiales y las instalaciones ( $72 \%)$ y menos horas de trabajo por jornada laboral $(20 \%)$. En conclusión, las soluciones propuestas por los docentes para reducir la incidencia de estas patologías son competencia de la Administración General del Estado y, por consiguiente, es su labor resolver esta situación para mejorar la seguridad y la salud de sus trabajadores.
\end{abstract}

Palabras clave: patologías, Educación Física, lugar de trabajo, salud laboral.

Abstract: The aim of the present study was to investigate Physical Education teacher's injuries related to their workplace conditions in the secondary schools from Jaen province, their effect in sick leaves, and the solutions suggested to enhance their health and quality of life. 50 teachers were randomly chosen from a sample of 216 instructors. All teachers filled in a standard questionnaire. 30\% suffered any injury in their workplace. The most common injuries suffered were: uncle sprain (13\%) and backache (10\%). These injuries caused 1 to 20 days long sick leaves. The solutions suggested by those Physical Education teachers were related to change teaching material and building conditions ( $72 \%)$, and to reduce the timetable (20\%). In conclusion and owing to legal regulations, General State Administration should improve Physical Education teacher's workplace conditions in order to keep their safety and health.

Key words:Physical Education, workplace conditions, safety, health.

\section{Introducción}

La problemática de los riesgos laborales es un temaque está captando la atención de los sectores de salud laboral en todos los países del mundo. Se han desarrollado estudios que analizan el efecto de los riesgos en diferentes ámbitos laborales y sus consecuencias físicas (Hämäläinen, Takala, \& Saarela, 2006; Rabadá \& Artazcoz, 2002; Takala, 1999), pero todavía queda mucho por aportar en el ámbito docente.

La docencia es una profesión dura que exige tratar con personas e implica la adaptación constante a nuevas necesidades en una sociedad cambiante (Rabadá \& Artazcoz, 2002). Debido a esta situación, los docentes están sometidos a un considerable grado de estrés, absentismo laboral y agotamiento (Calera, Esteve, Roel, \& Uberti-Bona, 2002). Pero además de estos riesgos psicosociales, existen otros de carácter físico y fisiológico que afectan en mayor o menor medida a los docentes dependiendo del área a la que estén vinculados. De acuerdo con el currículum para el área de Educación Física en Educación Secundaria Obligatoria (E.S.O.) (Real Decreto 116/2004, de 23 de enero, por el que se desarrolla la ordenación y se establece el currículo de la Educación Secundaria Obligatoria), la actividad docente del profesor de Educación Física, a diferencia de la de otras áreas, se caracteriza por la realización de un trabajo no sedentario. Esto puede estar relacionado con una serie de riesgos físicos derivados del ámbito laboral íntimamente ligados a las bajas laborales. El absentismo laboral por motivos de salud es un buen indicador tanto de la salud de las personas como de la salud de las organizaciones (Calera et al., 2002; Escalona, 2006).

De acuerdo con estas consideraciones y siguiendo las directrices del Ministerio de Sanidad y Consumo (MSC, 2000) y del Real Decreto

\footnotetext{
Fecha recepción: 16-04-09 - Fecha envío revisores: 17-04-09 - Fecha de aceptación: 07-06-09 Correspondencia: María Carrasco Poyatos

$\mathrm{C} /$ Argentina, $\mathrm{s} / \mathrm{n}$.

30720 Santigo de h Ribena (Murcia)

E-mail: mariacarrasco@um.es
}

487/1997 de 14 de abril, por el que se establecen las disposiciones mínimas de seguridad y salud relativas a la manipulación manual de cargas que entrañe riesgos, en particular dorso lumbares, para los trabajadores, se puede establecer que dentro de la actividad docente del profesor de Educación Física de E.S.O. las acciones más habituales que pueden estar vinculadas con patologías musculoesqueléticas y con la posibilidad de sufrir una baja laboral son la manipulación manual de cargas: es cotidiano levantar y transportar redes llenas de balones o balones medicinales o el empuje o la tracción de material gimnástico como potros o minitramps, o porterías, que por lo general tienen un peso mayor a $3 \mathrm{Kg}$ y pueden afectar seriamente a la salud laboral de estos trabajadores. El trabajo repetido del miembro superior, tronco y cuello, y el mantenimiento forzado de la postura en bipedestación son acciones que suelen manifestarse a diario en las clases debido a los propios requerimientos de las actividades físico-deportivas.

Otros riesgos físicos relacionados con patologías musculoesqueléticas y otro tipo de patologías que pueden producir bajas laborales están vinculados a las condiciones de los lugares de trabajo. De acuerdo con las consideraciones del Real Decreto 486/1997 de 14 de abril, por el que se establecen las disposiciones mínimas de seguridad y salud en los lugares de trabajo, éstas pueden estar relacionadas con las malas condiciones de limpieza y mantenimiento de las instalaciones deportivas, los cambios bruscos de temperatura si se desarrolla la sesión al aire libre, la falta de iluminación o el excesivo ruido.

España es uno de los países con mayor tasa de accidentes laborales del mundo (Hämäläinen et al., 2006), y se ha demostrado que los docentes en España sufren patologías físicas derivadas de su ámbito laboral (Preciado, Pérez, Calzada, \& Preciado, 2005), por tanto en el presente estudio se pretende mostrar las patologías más frecuentes que afectan a los profesores de Educación Física de E.S.O. de la provincia de Jaén, las bajas que habitualmente les mantienen alejados de su lugar de trabajo, y las soluciones que proponen para mejorar su situación laboral y a la vez su salud y su calidad de vida. 


\section{Material y método}

\subsection{Descripción de la muestra}

El universo de la muestra se formó por todos los profesores de Educación Física de Educación Secundaria Obligatoria que estuvieran impartiendo clases durante el curso 2004-2005 en la provincia de Jaén. Del total, colaboraron 216 docentes. De éstos, se seleccionó al azar una muestra representativa de 50 profesionales (a partir de: precisión del $5 \%$, prevalencia esperada del $3.5 \%$ y nivel de confianza del $95 \%$ ). Las características de los sujetos se muestran en la tabla 1.

\begin{tabular}{|c|c|c|c|}
\hline \multicolumn{4}{|c|}{ Tabla 1. Características de la muestra. } \\
\hline & & HOMBRES & MUJERES \\
\hline \multicolumn{2}{|c|}{$\mathrm{N}$} & 39 & 11 \\
\hline \multicolumn{2}{|c|}{ EDAD (años) } & 36.73 & 34.54 \\
\hline \multicolumn{2}{|c|}{ TIEMPO SER VICIO (años) } & 11.48 & 7.54 \\
\hline & Dipl. EF & 21 & 6 \\
\hline \multirow[t]{2}{*}{ TITULACIÓN } & Ldo. EF & 17 & 5 \\
\hline & Ldo.Med. & 1 & 0 \\
\hline
\end{tabular}

Dipl. EF: diplomado en Educación Física. Ldo. EF: licenciado en Educación Física. Ldo. Med: licenciado en Medicina.

\begin{tabular}{|c|c|l|}
\hline \multicolumn{3}{|c|}{ Tabla 2. Duración y frecuencia de las patologías sufridas por los docentes de Educación Física } \\
\hline DURACIÓN & FREC. & \multicolumn{1}{|c|}{ PATOLOGÍA } \\
\hline \multirow{4}{*}{$1-20$ días } & $10 \%$ & ESGUINCE DE TOBILLO. \\
\cline { 2 - 3 } & $9 \%$ & LUMBALGIA. \\
\cline { 2 - 3 } & $3 \%$ & FARINGITIS \\
\cline { 2 - 3 } & $3 \%$ & LESIONES ARTICULARES \\
\cline { 2 - 3 } & $3 \%$ & HERNIA DISCAL \\
\hline \multirow{4}{*}{$1-2$ meses } & $3 \%$ & ESGUINCE CERVICAL \\
\cline { 2 - 3 } & $3 \%$ & HERNIA DISCAL \\
\cline { 2 - 3 } & $3 \%$ & HERNIA INGUINAL \\
\hline \multirow{2}{*}{$>2$ meses } & $2 \%$ & AMENAZA PARTO PREMATURO \\
\cline { 2 - 3 } & $3 \%$ & ESGUINCE DE TOBILLO \\
\hline
\end{tabular}

\subsection{Instrumento de recogida de datos}

La recogida de datos se llevó a cabo mediante un cuestionario específico auto cumplimentado por los profesores. Debido a las dificultades a la hora de contar con un cuestionario «tipo» adecuado a las necesidades de investigación, se elaboró una encuesta original. En la elaboración definitiva del cuestionario se establecieron tres fases. En la primera se realizó un diseño provisional del mismo. En la segunda, se validó el cuestionario con un estudio piloto. Y en la tercera, se diseñó el cuestionario definitivo.

El cuestionario constó de 16 ítems con preguntas de carácter abierto y cerrado. Éstas se agruparon en: variables relacionadas con las características del profesorado, variables relacionadas con la docencia del profesorado, variables relacionadas con la percepción de ciertas cargas físicas en el desempeño de su trabajo, variables relacionadas con las bajas laborales que han sufrido estos trabajadores, variables relacionadas con las soluciones que se propondrían para mejorar su salud y su acción didáctica en las clases de Educación Física.

\subsection{Protocolo y temporización}

Antes de comenzar con la recogida de datos se solicitó a la Delegada de Educación de la provincia de Jaén su permiso para llevar a cabo el estudio. Tras obtenerlo, se contactó por teléfono con todos los centros deE.S.Ode la provincia de Jaén con la finalidad de solicitar la colaboración de los profesores implicados y explicarles su papel en el mismo. Los cuestionarios fueron cumplimentados de manera anónima y confidencial y reenviados por correo electrónico para su posterior análisis.

El procesamiento de los datos comenzó con la lectura y prueba de consistencia lógica de los cuestionarios para detectar aquellos datos que no cumplieran los requisitos exigidos o que presentaran inconsistencia o incoherencia en las respuestas. Esto dio paso la redacción de unas normas de codificación de las posibles respuestas y a la codificación de los cuestionarios en el programa estadístico Epi Info versión 3.2 para Windows. Tras la verificación de la codificación mediante la elección al azar de cuestionarios, se pasó al procesamiento estadístico de los datos con el programa estadístico Epi Info versión 3.2 para Windows y la redacción del informe final. Todo este proceso se llevó a cabo entre octubre de 2004 y junio de 2005.

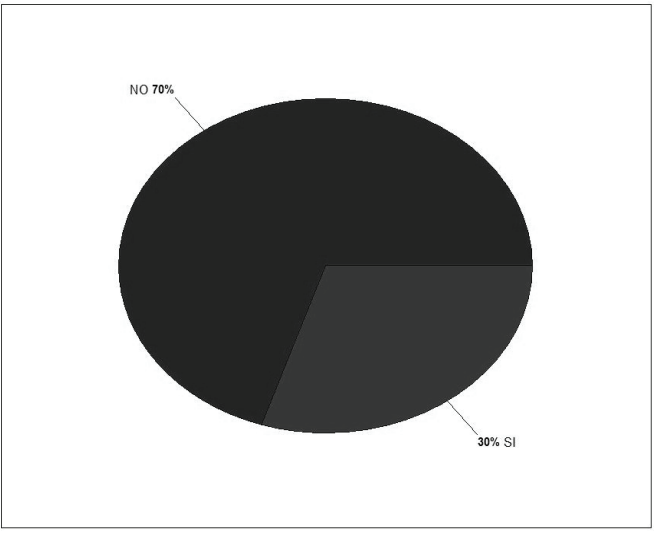

Figura 1. Porcentaje de docentes de Educación Fisica que han sufridopatologías físicas en su lugar de trabajo.

\subsection{Tratamiento de los datos}

Se utilizó el programa Epi Info, versión 3.2 para Windows del Center for Disease Control and Prevention (CDC). Se realizó un análisis descriptivo para las medias cualitativas y para las cuantitativas. Para comparar muestras de carácter cualitativo y conocer la posible relación entre variables cualitativas se utilizó el test de chi cuadrado. Para comparar muestras de carácter cuantitativo se utilizó el test de la t de Student (dos muestras) y el análisis de la varianza (más dos muestras). Para conocer la relación entre variables cuantitativas se utilizó el coeficiente de correlación $\mathrm{r}$ - de Pearson. Se estableció la significación estadística en $\mathrm{p}<0.05$.

\section{Resultados}

El 30\% de la población encuestada sufrió alguna vez una patología física en suámbito laboral (figura 1), viéndose los hombres más afectados (33.3\% del total) que las mujeres ( $18.18 \%$ del total) y siendo la población más afectada la correspondiente al grupo de edad de 31-40 años. Las patologías más frecuentes fueron: esguince de tobillo (13\%) y lumbalgia $(10 \%)$. Les siguen otras lesiones articulares y la hernia discal $(7 \%$ respectivamente). Las menos frecuentes fueron: hernia inguinal, faringitis, esguince cervical y parto prematuro (3\% respectivamente) (figura 2 ). Las mujeres parecieron verse más afectadas por otras lesiones articulares (9.09\%) y por el parto prematuro $(9.09 \%)$, mientras que las patologías que más predominaron en los hombres fueron el esguince de tobillo (30.77\%) y las lumbalgias (30.77\%), seguidas de la hernia discal (15.38\%) y el resto de patologías (7.69\%). No se encontraron relaciones significativas entre las patologías físicas sufridas y la edad y el género de los encuestados.

Las personas que se vieron afectadas por las patologías más frecuentes: esguince de tobillo y lumbalgia perciben en su lugar de trabajo ciertas cargas físicas negativas para su salud y su calidad de vida: cambios bruscos de temperatura ( $100 \%$ de los que padecieron esguince

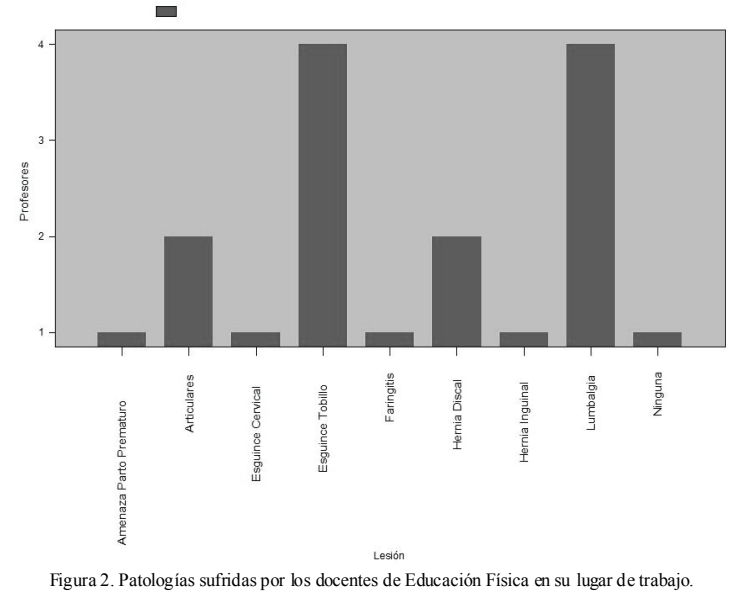


de tobillo y el $75 \%$ de los que padecieron lumbalgia), ruido excesivo ( $75 \%$ de ambos grupos), falta de higiene (100\% de los que padecieron esguince de tobillo y el $75 \%$ de los que padecieron lumbalgia), y el mantenimiento de la postura en bipedestación durante un tiempo prolongado ( $75 \%$ de los que padecieron lumbalgia). No se encontraron relaciones significativas entre las patologías físicas sufridas y las cargas físicas negativas del lugar de trabajo.

Para determinar la duración de las bajas por estas patologías físicas se hicieron 3 grupos. De 1-20 días duraron el 10\% de los esguinces de tobillo, el $9 \%$ de las lumbalgias y el $3 \%$ de las faringitis, hernia discal y lesiones articulares. De 1-2 meses, el 3\% de los esguinces cervicales, hernias discales y hernias inguinales. Más de 2 meses, $3 \%$ de los esguinces de tobillo. Estos resultados se detallan en la tabla 2.

Las soluciones que propusieron los profesores de Educación Física de E.S.O. del presente estudio para disminuir los efectos de las cargas físicas en su lugar de trabajo se resumen en las siguientes: mejorar los materiales y las instalaciones (72\%), menos horas de trabajo por jornada laboral (20\%). El 98\% de los encuestados considera que si se ponen en práctica estas medidas mejorará la salud y la calidad de vida de los docentes de esta área.

\section{Discusión}

De acuerdo con los datos obtenidos en el presente estudio, las patologías que más frecuentemente sufren los docentes de Educación Física deE.S.O. son de origen musculo-esquelético (esguinces de tobillo y lumbalgias). En relación a estos resultados existen coincidencias con los datos de otros trabajos previos realizados en centros docentes de diferentes provincias españolas (Guadalajara,Barcelona y Alicante), en los que se concluyó que las patologías más frecuentes que afectaron a los docentes y que además fueron motivo de baja laboral fueron las de origen musculo-esquelético, seguidas de las de origen psiquiátrico(Calera et al.,2002). En otro estudio realizado con docentes de La Rioja (Preciado et al., 2005), las patologías más frecuentes fueron las de origen psiquiátrico, seguidas de los trastornos de la voz y de las de origen musculo-esquelético, representadas en este caso por las lumbalgias y los esguinces principalmente. Debido a que en este estudio se incluyeron a docentes de todas las áreas y de todas las etapas educativas, no es de extrañar que las patologías musculo-esqueléticas no sean las más frecuentes, ya que el número de docentes de Educación Física en las diferentes etapas educativas es muy inferior al resto de docentes cuyo trabajo es fundamentalmente sedentario y, por tanto, no están tan vinculados a actividades físicas que puedan producir este tipo de patologías.

Parece ser, según los resultados del presente estudio, que las patologías más frecuentes son las que menos tiempo de baja laboral necesitan, manteniendo al profesorado fuera de su lugar de trabajo entre 1-20 días, excepto algún caso de esguince de tobillo más severo que ocasionó una baja laboral de más de 2 meses. Esto es un dato positivo, ya que indica que las patologías que sufren los docentes de Educación Física de E.S.O. son de carácter leve y tienen rápida solución.

Los profesores de Educación Física deE.S.O. que sufrieron esguinces de tobillo, relacionaron predominantemente sus patologías con los riegos físicos derivados de la calidad del ambiente interior de las instalaciones (temperatura, ruido, higiene), y no con el efecto de los riesgos físicos derivados de la manipulación manual de cargas o trabajo repetido del miembro superior, tronco y cuello, o el mantenimiento forzado de la postura. Aunque estos datos no fueron significativos, existe cierta relación con las estadísticas ofrecidas por The Annual Survey of Occupational Injuries and Iones (ASOII) (ASOII, 1998), en los que se indica que la mayoría de las patologías producidas por la manipulación manual de cargas afectaron a la parte superior de la espalda, en concreto, el $55 \%$ de estas patologías afectaron a la muñeca, el $7 \%$ a los hombros, y el $6 \%$ a la espalda. Del mismo modo, el Instituto Nacional de Seguridad e Higiene en el Trabajo (INSHT) (INSHT, 2000) indica que las bajas producidas por movimientos repetitivos estuvieron producidas por patologías musculo-esqueléticas de los miembros superiores, en concreto, Epicondilitis (29.3\%) y Tendinitis (26.7\%) localizadas en codos, manos, muñecas y hombros. No hay que olvidar que en la muestra del presente estudio se dieron casos de otras patologías relacionadas con los miembros superiores y la espalda (otras patologías articulares, hernia discal, esguince cervical) que según las consideraciones dispuestas anteriormente por el ASOII y el INSHT, pueden estar relacionadas con la manipulación manual de cargas y los movimientos repetitivos de los miembros superiores.

Por otro lado, los profesores de Educación Física de E.S.O. que sufrieron lumbalgias, relacionaron predominantemente sus patologías, además de con los riesgos físicos derivados de la calidad del ambiente interior de las instalaciones (temperatura, ruido, higiene), con el mantenimiento de la postura en bipedestación durante un tiempo prolongado. Aunque la relación entre estos resultados no fue significativa, es interesante indicar que, según el INSHT (INSHT, 2001), las posturas forzadas producen patologías músculo esqueléticas en músculos, tendones y nervios, que originan molestias, incomodidad, impedimento o dolor persistente.

Por último, hay que considerar que la mayoría de los profesores propone como soluciones a las patologías físicas sufridas en su lugar de trabajo mejorar los materiales e instalaciones y menos horas de trabajo porjornada laboral.Aun no siendo significativa la relación entre variables, se puede decir que si la jornada laboral fuera más corta, permanecerían menos tiempo en bipedestación y por tanto se podría reducir el riesgo de lumbalgias. Adicionalmente, la necesidad de carros para transportar materiales pesados, la construcción de gimnasios bien ventilados y amplios y la dotación de material variado podrían disminuir la incidencia de otras patologías articulares, hernia discal, esguince cervical o faringitis sufridas por la muestra del presente estudio.

Estos resultados sugieren que a pesar de la normativa vigente y de la obligatoriedad que tienen los poderes públicos de velar por la seguridad e higiene en el trabajo, todavía existen carencias en cuanto a determinados factores de riesgo físicos que pueden tener relación con las patologías que afectan a los docentes de Educación Física de E.S.O. Apesar de las limitaciones del presente estudio, se muestran unos resultados que la Administración General del Estado debería tener en cuenta y comprometerse a resolver en pro de la seguridad y la salud de sus trabajadores.

\section{Conclusiones}

Tras la intención del presente estudio de dar a conocer las patologías más frecuentes que afectan a los docentes de Educación Física de E.S.O. de la provincia de Jaén y el tiempo de baja laboral que se deriva de éstas incidiendo en su salud y su calidad de vida, se puede concluir que las patologías que más afectan a estos profesores son los esguinces de tobillo y las lumbalgias con una duración relativamente corta de entre 120 días de baja laboral. Las soluciones propuestas para reducir la incidencia de estas patologías son competencia de la Administración General del Estado y, por consiguiente, es su labor resolver esta situación para mejorar la seguridad y la salud de sus trabajadores.

\section{Agradecimientos}

A los profesores de Educación Física de E.S.O. de la provincia de Jaén, que amablemente se pusieron a nuestra disposición para colaborar en todo lo necesario. Ala Delegada de Educación de la provincia de Jaén por dar su permiso para que este estudio pudiera realizarse.

\section{Bibliografía}

ASOII.(1998). The nonfatal occupational injuries and illnesses involving days away from work by event or exposure leading to injury or illnes and selected parts of body affected by injury or illneses. Bureau of Labor Statistics, 6, 1-12. 
Calera, A. A., Esteve, L., Roel, J. M., \& Uberti-Bona, V. (2002). La salud laboral en el sector docente [Electronic Version]. www.bvsde.paho.org. Retrieved 23/11/2009,

Escalona, E. (2006). Relación salud-trabajo y desarrollo social: visión particular en los trabajadores de la educación. Revista Cubana de Salud Pública, 32(1), 1-19.

Hämäläinen, P., Takala, J., \& Saarela, K. L. (2006). Global estimates of occupational accidents. Safety Science, 44, 137-156.

INSHT (Ed.). (2000). Evaluación de los factores de riesgo de trastornos musculo-esqueléticos de la extremidad superior. Madrid: Centro Nacional de Nuevas Tecnologías.

INSHT (Ed.). (2001). La postura de trabajo. Madrid: Centro Nacional de Nuevas Tecnologías.

Real Decreto 116/2004, de 23 de enero, por el que se desarrolla la ordenación y se establece el currículo de la Educación Secundaria Obligatoria,

MSC. (2000). Protocolos de vigilancia sanitaria específica: movimientos repetidos del miembro superior. [Electronic Version]. Retrieved 23/ 11/2009,
Preciado, J., Pérez, C., Calzada, M., \& Preciado, P. (2005). Incidencia y prevalencia de los trastornos de la voz en el personal docente de La Rioja. Estudio clínico: cuestionario, examen de la función volcal, anállisis acústico y vídeolaringoestroboscopia. Acta Otorrinolaringologica Española, 56, 202-210.

Rabadá, I., \& Artazcoz, L. (2002). Identificación de los factores de riesgo laboral en docentes: un estudio Delphi.Archivos de Prevención de Riesgos Laborales, 5(2), 53-61.

Real Decreto 486/1997 de 14 de abril, por el que se establecen las disposiciones mínimas de seguridad y salud en los lugares de trabajo,

Real Decreto 487/1997 de 14 de abril, p. e. q. s. e. 1. d. m. d. s. y. s. r. a. 1.m.m.d.c. q.e.r., en particular dorso lumbares, para los trabajadores, Real Decreto 487/1997 de 14 de abril, por el que se establecen las disposiciones mínimas de seguridad y salud relativas a la manipulación manual de cargas que entrañe riesgos, en particular dorso lumbares, para los trabajadores.

Takala, J. (1999). Global estimates of fatal occupational accidents. Epidemiology, 10(5), 640-646.

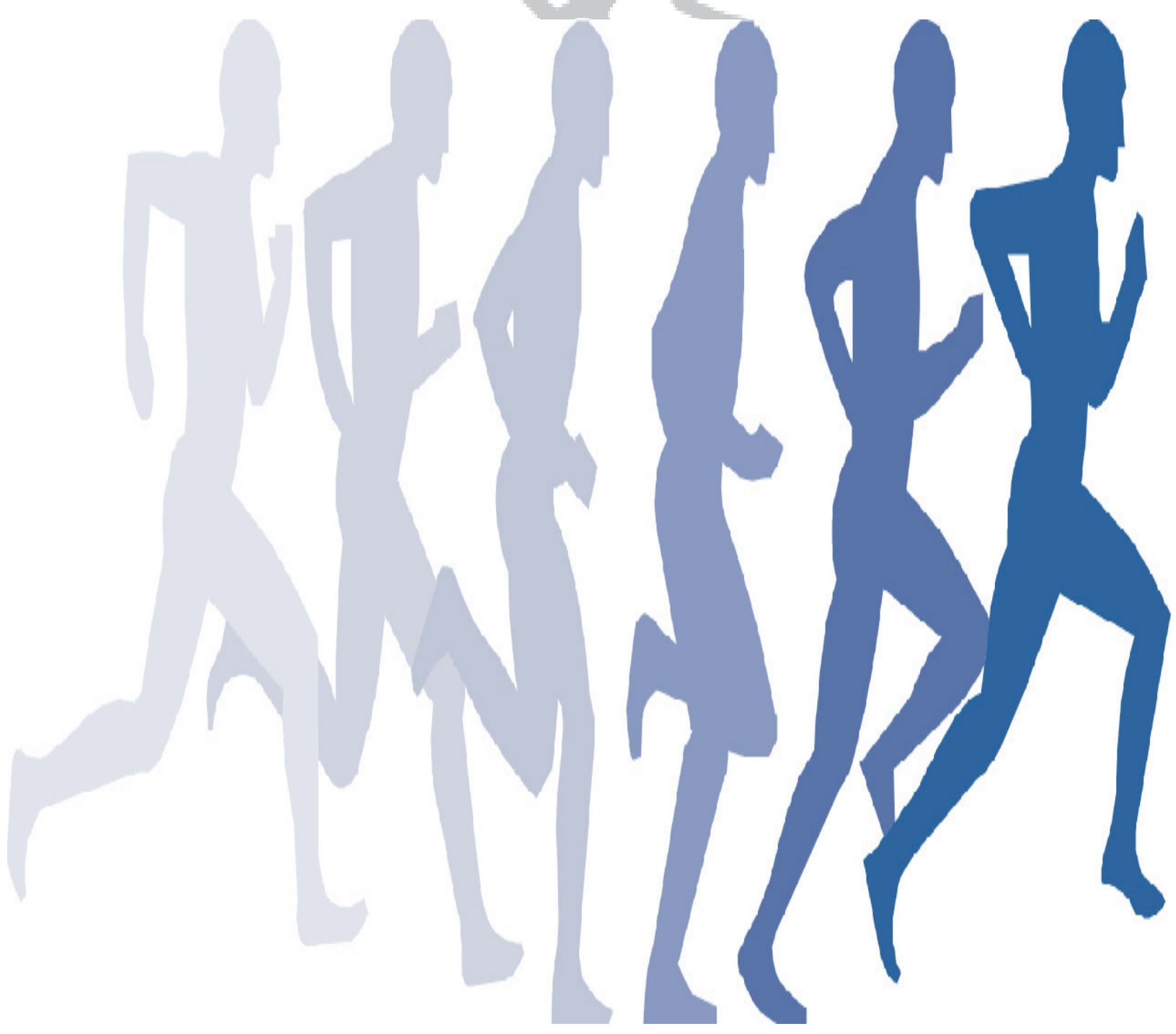

